\title{
Reabilitação no pós-cirúrgico de Síndrome do Impacto: estudo de
}

\section{caso}

A dor no ombro é uma das queixas mais comuns e incapacitantes do sistema musculoesquelético na população em geral. As lesões do manguito rotador, relacionadas à síndrome do impacto, são um grande fator de incapacitação crônica deste. O objetivo desse estudo foi avaliar a eficácia do tratamento fisioterapêutico no pós-cirúrgico da síndrome do impacto. Os parâmetros utilizados foram amplitude de movimento, quadro álgico e grau de força muscular. A pesquisa foi realizada na Clínica escola da Universidade Tiradentes, em Aracaju/SE, no período de Agosto à Outubro de 2010, realizaram-se 20 sessões com duração de 50 minutos cada. Os recursos utilizados foram: crioterapia, cinesioterapia e diatermia por ultra-som. Como resultados pôde-se observar a diminuição do quadro álgico, a melhora nas atividades de vida diária (AVD's) e melhora na amplitude de movimento (ADM), verificada através da goniometria. Concluiu-se que o tratamento fisioterapêutico mostrou-se eficaz na recuperação do pós-operatório do paciente com Síndrome do Impacto.

Palavras-chave: Síndrome do impacto; Fisioterapia; Ombro.

\section{Rehabilitation in the post-surgical Impact Syndrome: case study}

Shoulder pain is one of the most common and disabling complaints of the musculoskeletal system in the general population. The rotator cuff lesions, related to the impact syndrome, are a great factor of chronic incapacitation. The aim of this study was to evaluate the efficacy of the post-surgical physical therapy treatment of Impact Syndrome. The parameters used were range of motion, pain chart and degree of muscular strength. The research was carried out at the School Clinic of the Tiradentes University, in Aracaju / SE, from August to October 2010, with 20 sessions lasting 50 minutes each. The resources used were: cryotherapy, kinesiotherapy and diathermy by ultrasound. As a result, it was possible to observe a decrease in pain, improvement in activities of daily living (ADL) and improvement in range of motion (WMD), verified through goniometry. It was concluded that the physical therapy treatment was effective in the postoperative recovery of the patient with Impact Syndrome.

Keywords: Impingement Syndrome; Physiotherapy; Sholder.

Topic: Fisioterapia

Reviewed anonymously in the process of blind peer.
Received: $13 / 11 / 2018$

Approved: $24 / 01 / 2018$
Maria Gabriela Reis Santos (D)

Universidade Tiradentes, Brasil

http://lattes.cnpq.br/5055994764208732

http://orcid.org/0000-0003-0804-5409

gaabifisio@hotmail.com

Genildo Aragão Junior

Universidade Tiradentes, Brasil

http://lattes.cnpq.br/8438187134371321

http://orcid.org/0000-0001-8793-3361

genildojr fisio@hotmail.com

Edna Aragão Farias Cândido (ic

Universidade Tiradentes, Brasil

http://lattes.cnpq.br/8385700783273687

http://orcid.org/0000-0002-9441-0697

edna aragao1@globo.com
Tássia Virginia de Carvalho Oliveira

Universidade Tiradentes, Brasil

http://lattes.cnpq.br/2273361044731342

tassinhafisio@yahoo.com.br

\section{Referencing this:}

SANTOS, M. G. R.; ARAGÃO JUNIOR, G.; CÂNDIDO, E. A. F.; OLIVEIRA, T. V. C.. Reabilitação no pós-cirúrgico de Síndrome do Impacto: estudo de caso. Scire Salutis, v.8, n.1, p.8-15, 2019. DOI: http://doi.org/10.6008/CBPC2236-9600.2018.001.0002

DOI: 10.6008/CBPC2236-9600.2018.001.0002 


\section{INTRODUÇÃO}

A dor no ombro é uma das queixas mais comuns e incapacitantes do sistema musculoesquelético na população em geral (POPE et al., 2001). Apresenta prevalência estimada entre $15 \%$ a $25 \%$ nos pacientes que procuram clínicas ortopédicas e de fisioterapia (LECLER et al., 2004), entre as várias causas que podem levar ao surgimento desse sintoma, estão às lesões do manguito rotador relacionadas à síndrome do impacto, podendo acometer pacientes de todas as faixas etárias e sem preferência por algum sexo ou raça, sendo a mais frequente causa de incapacitação crônica do ombro (MICHAEL et al., 2004).

O ombro é uma articulação bastante complexa e a mais móvel de todo o corpo humano, entretanto é considerada pouco estável por sua anatomia articular, especialmente na articulação glenoumeral. Esta grande mobilidade e menor estabilidade podem ser atribuídas à frouxidão capsular associada à forma arredondada e grande da cabeça umeral e rasa superfície da fossa glenóide (SANTOS et al., 1995), sendo necessária harmonia sincrônica e constante entre todas as estruturas estáticas e dinâmicas que mantêm sua biomecânica normal. Por esse motivo, qualquer alteração que comprometa sua estrutura e função faz com que esse complexo articular seja alvo de inúmeras afeç̧ões, sendo a síndrome do impacto (SI) a mais comum em indivíduos adultos. Esta patologia caracteriza-se por uma síndrome dolorosa do ombro, normalmente acompanhada por microtraumatismos e degeneração, além do déficit de força muscular e tendinite do manguito rotador (MOREIRA et al., 1998).

O manguito rotador (MR) é formado posteriormente pelos tendões dos músculos supraespinal (Se), infraespinal (le), redondo menor $(\mathrm{Rm})$ e, anteriormente, pelo tendão do músculo subescapular $(\mathrm{Sc})$, tendo contribuição importante nas funções de mobilidade e estabilidade do ombro. Lesões desta estrutura representam uma das maiores causas de incapacidade no ombro (MURRAY et al., 2002)

Em 1972, Neer individualizou a patologia Síndrome do Impacto no Ombro (S.I.O.), descrevendo sua fisiopatologia e quadro clínico em seus diferentes estágios, bem como as propostas terapêuticas e cirúrgicas. Porém, foi em 1788, que a primeira ruptura do manguito rotador foi descrita, por Monro (MOREIRA et al., 1998), embora alguns autores defendam que a primeira ruptura do manguito tenha sido descrita por Smith, em 1834 (ANDREWS, 2000).

As roturas do manguito rotador (MR) são frequentes na população acima dos 50 anos de idade, sendo uma doença de etiologia multifatorial, com característica de lesão degenerativa na maioria dos casos, incidindo em 10 a 90\% da população (WILLIAMS, 2004; VEADO, 2006). O diagnóstico está fundamentado na avaliação clínica e por métodos de imagem. Fatores como idade fisiológica e demanda funcional determinarão se o tratamento será conservador ou cirúrgico, reparo aberto ou artroscópico (GODINHO et al., 2010).

O impacto ou compressão é a invasão do acrômio, do ligamento coracoacromial, do processo coracóide ou da articulação acromioclavicular sobre o mecanismo do manguito rotador que passa por de baixo deles quando a articulação glenoumeral é movimentada, particularmente em flexão e rotação interna, mas esse impacto provoca inflamações no tendão (tendinite) ou na bursa (bursite) (ANDREWS, 2000). 
Não há comprovação se os mecanismos extrínsecos (impacto subacromial primário, instabilidade glenoumeral, impacto com o processo coracóide, impacto com o rebordo póstero-superior da glenóide) representam o evento primário responsável pela tendinite do manguito rotador ou se a compressão desse manguito ocorre devido a uma doença intrínseca (fraqueza muscular, uso excessivo, hipovascularização tendínea) a estes músculos (VEADO et al., 2006).

A síndrome do impacto é dividida em três fases: fase I - dor aguda e início súbito, de tratamento exclusivamente conservador; fase II - caracterizada pela dor crônica e resposta favorável ao tratamento conservador, em $70 \%$ dos casos, e subdividida em fases IIA - sem ruptura de tendão, e IIB - com ruptura parcial; fase III - ruptura completa de um ou mais tendões, frequentemente requerendo tratamento cirúrgico (GODINHO et al., 1995).

O tratamento da síndrome do impacto é baseado na reabilitação, pelo menos por três a seis meses; na falha deste, pode-se optar pelas diversas técnicas cirúrgicas. Dentre essas há a artroscopia, que na última década deixou de ser utilizada apenas para diagnóstico e tornou-se fundamental na descompressão subacromial, reparo de lesão do manguito rotador e no tratamento da artrose sintomática da articulação acromioclavicular (DONEUX et al., 1998). O tratamento artroscópico das lesões extensas do manguito rotador leva a $89 \%$ de excelentes e bons resultados e proporciona aumento da amplitude do movimento do ombro (MIYAZAKI et al., 2009).

A reabilitação da articulação do ombro pode ser difícil, não só pela sua função complexa que envolve a integridade anatômica e funcional, mas também devido a contribuições fisiológicas e biomecânicas das estruturas como, por exemplo, da escápula. De forma geral, programas de reabilitação do ombro empregam, na maior parte dos casos, exercícios com cargas e intensidades progressivas de acordo com o tipo de lesão e procedimento cirúrgico realizado (KIBLER, 2001;). Ainda que essas características sejam determinantes na progressão dos exercícios, o conhecimento da mecânica articular do ombro é fundamental para a escolha adequada dos exercícios (HAYES et al., 2004).

\section{METODOLOGIA}

\section{Amostra}

Sendo este estudo de caso, de caráter interventivo, com abordagem qualitativa prospectiva, assim, ele foi realizado na clínica escola da Universidade Tiradentes no período de Agosto à Outubro de 2010, com paciente do sexo masculino, 43 anos de idade, da raça negra, diagnóstico clínico de síndrome do impacto e diagnóstico cinético funcional de limitação moderada para AVD's. Durante a anamnese relatou que fez a cirurgia há três semanas e desde então não realizou tratamento fisioterapêutico, queixa-se de quadro álgico em forma de pontada. Apresenta ao exame físico dois pontos de cicatriz cirúrgica na região superior e medial do ombro direito; na palpação foram encontrados pontos de tensão no bíceps braquial, deltoide médio, trapézio superior e peitoral maior. Presença de edemas no membro examinado, textura e hidratação da pele adequada, possui postura antálgica com ombro direito em rotação interna e adução de ombro, flexão de 
cotovelo e pronação de antebraço. Não faz uso de órteses ou próteses, realiza abdução do ombro em bloco. Apresenta grau seis (6) de dor, de acordo com a Escala Visual Analógica da Dor (EVAD) e apresenta crepitações na articulação. Segundo a formalidade prescrita, após esclarecimentos devidos, houve concordância do paciente em participar da pesquisa, assinando o Termo de Consentimento Livre e Esclarecido.

\section{Instrumentos}

$\mathrm{Na}$ avaliação foi utilizado o goniômetro manual para mensurar a amplitude de movimento do paciente; testes clínicos (Hawkins; Jobe; Neer; Palm-up; Patte; Apley; Yergason; e Gerber) para avaliar a integridade articular do ombro; teste de força muscular e Escala Visual Analógica da Dor (EVAD). Os testes clínicos são fundamentais para examinar uma articulação de forma completa e com grande acurácia, é uma parte crítica do processo de diagnóstico para o examinador (WEINSTEIN et al., 2000).

A goniometria é realizada através do goniômetro universal, sendo formado por dois braços e um eixo, um braço vai acompanhar o movimento, o outro vai permanecer fixo até o final da medida, e o eixo vai ficar sobre a articulação avaliada. Tem a vantagem de ser um instrumento barato, fácil de manusear e as medidas são tomadas rapidamente (SACCO et al., 2007). A goniometria, teste de força muscular e os testes clínicos foram realizados por um fisioterapeuta com o paciente sem camisa sentado na maca.

A EVAD consiste de uma reta graduada de 0 a 10, onde zero significa ausência de dor e dez, dor insuportável, logo, quanto maior o escore, maior a intensidade da dor. O paciente foi instruído a marcar um número que indicava a intensidade da dor que sentia. $\mathrm{O}$ teste de força muscular destina-se a avaliar a capacidade de o músculo desenvolver tensão contra uma resistência. É um método subjetivo, porém muito utilizado na avaliação clínica, possuindo cinco graus de forma crescente, sendo que Grau zero é ausência de contração muscular e Grau cinco é capacidade de vencer uma força máxima (NICHOLAS et al., 1977).

\section{Procedimentos}

Foram realizadas vinte sessões para a reabilitação do paciente, sendo três atendimentos semanais com duração de 50 minutos cada. 0 objetivo inicial do tratamento era analgesia e ganho de mobilização intra-articular, os objetivos a médio prazo eram ganho de amplitude de movimento e fortalecimento muscular. Para estes objetivos foram utilizados: Ultra-Som de forma contínua, com $1 \mathrm{MHz}$ e duração de seis minutos; mobilização passiva grau 3 e 4 da escápula, de forma látero-lateral e crânio-caudal e da cabeça umeral de forma ântero-posterior e crânio-caudal; alongamento passivo mantido com duração de trinta segundos da musculatura do trapézio (superior, médio e inferior), peitoral maior e deltoide (fibras anterior e media); fortalecimento de forma isotônica em cadeia cinética fechada dos músculos do deltoide médio e manguito rotador (Sub-escular, supra e infra espinhoso e redondo menor), através dos acessórios da mecanoterapia: mecano-plus, theraband e halteres; massagem transversa (Cyriax) no bíceps braquial, trapézio superior, deltoide médio e peitoral maior, e propriocepção através de movimentos angulares, 
usando uma bola suíça de $65 \mathrm{~cm}$, com o ombro a 90, empurrando a bola contra a parede. Ao final de cada uma das vinte sessões foi realizado crioterapia, aplicando bolsas de gelo diretamente na articulação, com duração de vinte minutos.

\section{RESULTADOS}

Os resultados obtidos foram aqui descritos por meio da avaliação da goniometria, grau de contração muscular e testes especiais. Esses parâmetros foram realizados antes e após as 20(vinte) sessões. Os resultados obtidos antes das 20(vinte) sessões estão descritos na Tabela 1 e 2 e os que foram obtidos depois das 20(vinte) sessões estão expostos na Tabela 3 e 4.

Tabela 1: Goniometria ativa e passiva realizada pelo Fisioterapeuta.

\begin{tabular}{|c|c|c|c|c|c|c|}
\hline GONIOMETRIA & Flexão & Extensão & Abdução & Adução & Rot. Ext. & Rot. Int. \\
\hline Ativa & 80 응 & 240 & 880 & 20 응 & 140 & 500 \\
\hline Passiva & $110^{\circ}$ & 460 & $120^{\circ}$ & 40 은 & $20 \circ$ & 640 \\
\hline
\end{tabular}

Tabela 2: Testes Clínicos realizados pelo Fisioterapeuta.

\begin{tabular}{|l|l|l|}
\hline TESTES CLÍNICOS & Positivo & Negativo \\
\hline Hawkins & $\mathrm{X}$ & \\
\hline Jobe & $\mathrm{X}$ & \\
\hline Neer & $\mathrm{X}$ & \\
\hline Palm Up & $\mathrm{X}$ & $\mathrm{X}$ \\
\hline Patte & & \\
\hline Apley & $\mathrm{X}$ & \\
\hline Yergason & $\mathrm{X}$ & $\mathrm{X}$ \\
\hline Gerber & & \\
\hline
\end{tabular}

Grau de força muscular: Grau 4 dos músculos flexores, extensores e adutores do ombro, e grau 3 para rotadores externos e internos e abdutores no MSD e grau 5 para todos os movimentos do MSE.

Tabela 3: Goniometria ativa e passiva realizado pelo Fisioterapeuta.

\begin{tabular}{|c|c|c|c|c|c|c|}
\hline GONIOMETRIA & Flexão & Extensão & Abdução & Adução & Rot. Ext. & Rot. Int. \\
\hline Ativa & $156^{\circ}$ & 40 & 1600 & 20 & 70 잉 & 780 \\
\hline Passiva & $180^{\circ}$ & 460 & $178^{\circ}$ & $40^{\circ}$ & 780 & $84^{\circ}$ \\
\hline
\end{tabular}

Tabela 4: Goniometria ativa e passiva realizado pelo Fisioterapeuta.

\begin{tabular}{|l|l|l|}
\hline TESTES CLÍNICOS & Positivo & Negativo \\
\hline Hawkins & & $\mathrm{X}$ \\
\hline Jobe & & $\mathrm{X}$ \\
\hline Neer & $\mathrm{X}$ & \\
\hline Palm Up & & $\mathrm{X}$ \\
\hline Patte & & $\mathrm{X}$ \\
\hline Apley & & $\mathrm{X}$ \\
\hline Yergason & & $\mathrm{X}$ \\
\hline Gerber & & $\mathrm{X}$ \\
\hline
\end{tabular}

Grau de força muscular: Grau cinco para todos os movimentos do MSE e do MSD.

Os resultados obtidos foram analisados de forma qualitativa, por meio de relatos do paciente ao afirmar a redução do grau de dor (de grau 6 para grau 1) através da EVAD, a sua maior satisfação e capacidade de realizar suas atividades de vida diária e de forma quantitativa por meio da avaliação da força muscular, encontrando-se ao final do tratamento em grau cinco. A evolução na amplitude de movimento pode ser vista na comparação da goniometria, antes e após da intervenção fisioterapêutica. Os testes clínicos também apresentaram alterações significativas, caracterizando um bom posicionamento articular e preservação da 
boa função, persistiu a positividade no teste de Neer, caracterizando o impacto entre o músculo supra espinhoso com a borda inferior do acrômio.

\section{DISCUSSÃO}

O maior benefício do frio durante a reabilitação é a diminuição da dor e do espasmo muscular, permitindo, assim, a mobilização precoce (KISNER et al., 2001). Neste estudo a analgesia era realizada, em curto prazo, através da crioterapia, assim os resultados foram muito significativos e corroboram com os estudos de Donegar (DONEGAR et al., 2002), ao afirmar que o resfriamento favorece a redução da dor e do processo inflamatório.

A mobilização era realizada no limite da amplitude de movimento do paciente, sendo realizada antes da mesma, uma mobilização escapular nas direções látero-lateral e crânio-caudal. Mobilização é usada para alongar ou liberar com segurança determinada estrutura de modo a restaurar a mecânica articular normal (KISNER et al., 2001). Resultados semelhantes a este estudo foram encontrados por Barbosa (BARBOSA et al., 2008), ao adicionar técnicas de mobilização articular ao tratamento das tendinites do ombro e encontrar importantes ganhos funcionais.

Azevedo (2008) mostra que a utilização de exercícios de alongamento tem sido efetiva no aumento da amplitude de movimento (ADM) em diferentes articulações. Clinicamente, estes exercícios são prescritos em condições de grande limitação de ADM, como em períodos pós-imobilização; assim como em pequenas limitações de ADM observadas em indivíduos saudáveis, o que também se observa neste estudo.

A massagem transversa, realizada no paciente era de grande importância para o processo de reabilitação, pois ela se torna mais importante ainda quando associada à utilização do ultra-som, o que era existente no percurso desse caso, assim, no paciente, proporcionou uma melhor função da articulação do ombro e por consequência depressão da dor e ganho da amplitude de movimento. A massagem por friç̧ão tem a finalidade de mobilizar os músculos e liberar as fibras tendinosas, os músculos ou tecidos cicatriciais, as aderências que restrinjam o movimento que causam dor, portanto essa técnica é utilizada para facilitar a irrigação sanguínea local (STARKEY et al., 2001). A associação de calor profundo (ultra-som) e massagem é de grande valia para manter a cicatriz cirúrgica móvel e indolor (VEADO et al., 2006).

O paciente também apresentou grandes avanços em se tratando do fortalecimento muscular, logo as técnicas utilizadas corresponderam aos objetivos do tratamento. A reabilitação desse tipo de lesão deve concentrar-se em desenvolver a estabilidade articular dinâmica, assim os exercícios devem tentar fortalecer os músculos do manguito rotador para estabilizar dinamicamente a articulação glenoumeral e os estabilizadores escapulares que ajudam a orientar a fossa glenóide com a cabeça do úmero, a fim de preservar a estabilidade e ajudar a prevenir os problemas do ombro (ANDREWS, 2000). O trabalho de Soares (SOARES ET AL., 2003) também demonstra a importância do fortalecimento muscular para prevenção de lesões em atletas de judô ao mostrar o ganho de força e estabilidade com o trabalho de fortalecimento muscular precoce. 
A propriocepção teve uma grande importância no tratamento, devido às suas finalidades, visto que, as mesmas interferem totalmente na mecânica da articulação, promovendo uma melhor evolução no quadro do paciente. Consciência postural do movimento e das mudanças de equilíbrio, assim como o conhecimento da posição do peso e da resistência de objeto ao corpo, além de sensações de movimento articular e de posição articular, assim é definida a propriocepção (VOLPON et al., 1997). O estudo de Antunha (ANTUNHA et al., 2008) também ressalta a importância da propriocepção para a reabilitação na medida em que a propriocepção prepara a articulação para resistir a possíveis perturbações futuras sem sofrer lesão.

\section{CONCLUSÕES}

O ombro está sujeito a uma rápida instalação de um quadro de rigidez e atrofia muscular no período pós-operatório, e devido a sua importante função de constantemente equilibrar duas forças, aparentemente incompatíveis: mobilidade e estabilidade, se faz importante a reabilitação nessa fase. Desta forma, o objetivo desse estudo de caso, o qual era mostrar o efeito do tratamento fisioterapêutico no pós-cirúrgico da Síndrome do Impacto, através da aplicação de um protocolo de reabilitação baseado em diatermia por ultrasom, crioterapia e cinesioterapia, e após os dados aqui obtidos, concluiu-se que o tratamento fisioterapêutico mostrou-se eficaz na recuperação do pós-operatório do paciente com Síndrome do Impacto, levando-o ao retorno precoce as suas atividades de vida diária.

\section{REFERÊNCIAS}

ANDREWS, J.. Reabilitação física das lesões desportivas. 2 ed. Rio de Janeiro: Guanabara Koogan, 2000.

ANTUNHA, E. L. G.; SAMPAIO, P.. Propriocepção: um conceito de vanguarda na área diagnóstica e terapêutica. Acad. Paul. Psicol, São Paulo, v.28, n.2, p.278-283, 2008.

AZEVEDO, D. C. C.; SILVIA, C. D. L.; ELISA, W. P. S.; SARA, P. D.; LOUREIRO, M.. Influência da limitação da amplitude de movimento sobre a melhora da flexibilidade do ombro após um treino de seis semanas. Revista brasileira de medicina do esporte, v.14, n.2, p.119-121, 2008.

BARBOSA, R. I.; GOES, R.; MAZZER, N.; FONSECA, M. C. R.. A influência da mobilização articular nas tendinopatias dos músculos bíceps braquial e supra-espinhal. Revista Brasileira de Fisioterapia, São Carlos, v.12, n.4, p.298-303, 2008.

DONEGAR, C. R.; DONLEY, P. B.. Controle da dor com as modalidades terapêuticas. In: PRENTICE, W. E.. Modalidades terapêuticas em medicina esportiva. São Paulo, 2002. p.1937.

DONEUX, S. P.. Incidência de dor acromioclavicular após descompresssäo subacromial artroscópica. Revista brasileira de ortopedia, v.33, n.5, p.329-32, 1998.

GODINHO, G. G.. Artroscopia cirúrgica no tratamento da síndrome do impacto: nossa experiência em 100 casos cirúrgicos. Revista Brasileira de Ortopedia, p.540-546, 1995.

GODINHO, G. G.. Avaliação da integridade anatômica por exame de ultrassom e funcional pelo índice de Constant \&
Murley do manguito rotador após reparo artroscópico. Revista brasileira de ortopedia, v.45, n.2, p.174-180, 2010.

HAYES, K.; GINN. K. A.; WALTON, J. R.; SZOMOR, Z. L.; MURRELL, G. A. C.. A randomized clinical trial evaluating the efficacy of physiotherapy after rotator cuff repair. Australian Journal of Physiotherapy, v.50, p.77-83, 2004.

KIBLER, W. B.; MCMULLEN, J.; UHL, T.. Shoulder rehabilitation strategies, guidelines and practice. Orthopedic Clinics of North America, v.32, n.3, p.527-38, 2001.

KISNER, C.; COLBY, L. A.. Exercícios terapêuticos: fundamentos e técnicas. São Paulo: Manole, 2001.

LECLER, A.; CHASTANG, J. F.; LANDRE, M. F.; ROQUELAURE, $Y$.. Incidence of shoulder pain in repetitive work. Occupatinal and Environmental Medicine, v.61, p.39-44, 2004.

MICHAEL, S.; OSVANDRE, L.. O tratamento cirúrgico da síndrome do impacto idiopática no processo coracóide. Revista brasileira de ortopedia, v.34, p.23-26, 2004.

MIYAZAKI, A. N.; FREGONEZE, M.; SANTOS, P. D.; SILVA, L. A.; ORTIZ, E. C. M. M. P. R. T.; CHECCHIA, S. L.. Lesões extensas do manguito rotador: avaliação dos resultados do reparo artroscópico. Revista Brasileira de Ortopedia, v.44, n.2, p. 148-152, 2009. DOI: http://dx.doi.org/10.1590/S0102$\underline{36162009000200010}$

MOREIRA, C.; CARVALHO, M. A. P.. Noções práticas de reumatologia. Belo Horizonte: Health, 1998. 
MURRAY, T. F.; LAJTAI, G.; MILESKI, R. M.; SNYDER, S. J.. Arthroscopic Repair of medium to large full-thickness rotator cuff tears: Outcome at 2-6-year follow-up. J Shoulder Elbow Surg, v. 11, p.19-24, 2002.

NICHOLAS, J. A.; SAPEGA, A.; KRAUS, H.; WEBB, J. N.. Factors influencing manual muscle tests in physical therapy. J Bone Joint Surg Am, Las Vegas, v.60, n.2, p.186-90, 1978.

POPE, D. P.; SILMAN, A. J.; CHERRY, N. M.; PRITCHARD, C.; MACFARLANE, G. J.. Association of occupational physical demands and psychosocial working environment with disabling shoulder pain. Annals of the Rheumatic Diseases, v.60, n.9, p.852-858, 2001.

SACCO, I. C. N.; ALIBERT, S.; QUEIROZ, B. W. C.; PRIPAS, D.; KIELING, I.; KIMURA, A. A.; SELLMER, A. E.; MALVESTIO R. A.; SERA, M. T.. Confiabilidade da fotogrametria em relação à goniometria para avaliação postural de membros inferiores. Revista Brasileira de Fisioterapia, São Carlos, v.11, n.5, p.411-417, 2007.

SANTOS, O. S.; BONAMIC, C.; SOBANIA, L. C.; OTSUKA, N.; SOBANIA, R. L.. Síndrome do impacto: resultados do tratamento cirúrgico. Revista Brasileira de Ortopopedia, v.30, n.9, p.655-6559, 1995.

SOARES, S. T. M.. Trabalho preventivo para lesões de ombro e cintura escapular em atletas amadores de judô. Revista brasileira de ciência e movimento, v.11, n.1, p.29-33, 2003.

STARKEY, C. H. A. D.. Recursos terapêuticos em fisioterapia. São Paulo: Manole, 2001.

VEADO, M. A. C.; GOMES, T. P. O.; PINTO, R. Z. A.. Análise funcional e estrutural do reparo das lesões extensas do manguito rotador. Revista Brasileira de Ortopedia, v.41, n.8, p.294-301, 2006.

VOLPON, J. B.; PRADO, M. R. A.. Vascularização do manguito rotador do rato. Revista Brasileira de Ortopedia, v.32, p.699-702, 1997.

WEINSTEIN, S. L.; BUCKWALTER, J. A.. Ortopedia de Turek: Princípios e sua Aplicação. 5 ed. São Paulo: Manole, 2000.

WILLIAMS, G. R. J. R.; ROCKWOOD, C. A. J. R.; BIGLIAN, I.; IANNOTI, J. P.; STANWOO, W.. Rotator cuff tears: Why do we repair them?. J Bone Joint Surg Am, v. 86, n.12, p.2764-76, 2004.

A CBPC - Companhia Brasileira de Produção Científica (CNPJ: 11.221.422/0001-03) detém os direitos materiais desta publicação. Os direitos referem-se à publicação do trabalho em qualquer parte do mundo, incluindo os direitos às renovações, expansões e disseminações da contribuição, bem como outros direitos subsidiários. Todos os trabalhos publicados eletronicamente poderão posteriormente ser publicados em coletâneas impressas sob coordenação da Sustenere Publishing, da Companhia Brasileira de Produção Científica e seus parceiros autorizados. Os (as) autores (as) preservam os direitos autorais, mas não têm permissão para a publicação da contribuição em outro meio, impresso ou digital, em português ou em tradução. 